\title{
Numerical Simulation of Aerated Powder Consolidation ${ }^{1}$
}

\author{
Kristy A. Coffey and Pierre A. Gremaud \\ Department of Mathematics and Center for Research in Scientific Computation, North Carolina State \\ University, Raleigh, NC 27695-8205, USA
}

\begin{abstract}
When a fine powder is dumped into a silo, the gas trapped by the particles will slowly escape by diffusing through the material. The corresponding uneven gas pressure distribution creates a body force that is taken into account through Darcy's law. By using spatial averaging, the formulation, even though essentially one-dimensional in space, includes effects due the geometry of the container. An efficient and robust numerical scheme based on a DAE formulation is proposed and implemented. Various computational results are presented and discussed to establish the validity of the approach.
\end{abstract}

\section{INTRODUCTION}

This paper deals with various problems related to the simulation of aerated powder consolidation. This kind of phenomena is routinely encountered during the handling of fine powders in countless applications. Typically a powder is stored in a bunker or silo, see Figure 1. During filling, air gets trapped in the material leading, in some cases, to partial fluidization [8], [14] and noticeable changes of the mechanical properties. Over time the excess air diffuses through the powder and eventually escapes through the top surface. This paper aims to find the length of time a given material takes to consolidate. With respect to applications, the ultimate goal is to be able to predict and consequently avoid flooding, i.e., the sudden discharge from a hopper of a fine powder at a much greater rate than that of the flow of ordinary granular materials. Further comments on the connection between flooding and deaeration can be found in [12].

Early work by Janssen [6] analyzed the behavior of a column of granular material in a container. Forces due to the gradient of gas pressure were neglected, and the analysis was restricted to vertical cylinders. Some other models are based on drastic simplifications, such as considering a constant vertical stress [9].

The present model is derived from basic conservation principles of mass and momentum. The forces resulting from nonuniform gas pressure are taken into account through Darcy's law. The modeling assumptions roughly follow that of [8], see also [4], [12]. In those publications, the effects of the geometry of the container are neglected, in effect, treating only the case of cylindrical bunkers. In [4], that case is mathematically analyzed, and a

\footnotetext{
${ }^{1}$ This project was supported by the Army Research Office (ARO) through grant DAAD19-99-1-0188. The first author was partially supported by a Department of Education GAANN Fellowship. The second author was partially supported by the National Science Foundation (NSF) through grant DMS- 9818900.
} 


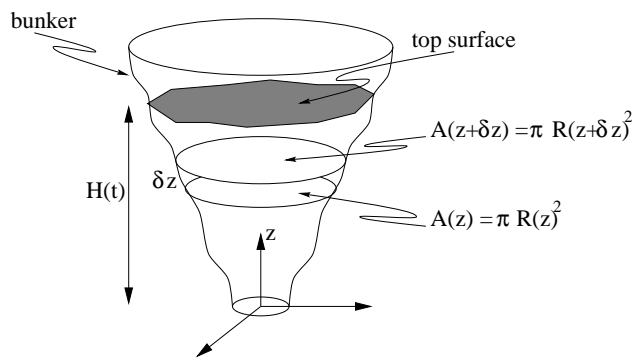

FIG. 1. Geometry and coordinate systems for the vertical conical bunker. The height of the column of powder of time $t$ is denoted $H(t)$.

robust numerical method is proposed and implemented. One of the contributions of this paper is the extension of those models to general axisymmetric domains. Although the modification may appear as a technical detail, it has profound ramifications with respect to the structure of the system, see remarks at the end of $\S 2$.

Apart from using specific physical constitutive laws, the main restrictive assumption consists of neglecting the fluctuations in the horizontal directions, allowing for an essentially one dimensional in space formulation. In other words, the problem is described exclusively in terms of quantities that have been averaged in the horizontal direction. It should be noticed that in many situations the use of quasi one-dimensional consolidation models can only be viewed as a first step, and full multidimensional approaches should be considered instead. We refer to [16] and [10], Chap.5, for remarks about the limitations of such models. More importantly however, even a simplified model such as the one considered here is very sensitive to the values of various material coefficients such as compressibility. This is clearly illustrated by our numerical results, see e.g. Figure 5. Those material coefficients are typically hard to measure in an accurate way.

The paper is organized as follows. The model is derived in Section 2. The resulting system is nonlinear and strongly coupled. It consists of an essentially parabolic PDE, an ODE and an integral equation. The three main unknowns are the average vertical stress, the average gas pressure and the height of the powder in the container. Through an appropriate transformation, the calculations are performed in a fixed reference computational domain. A discretization is proposed in Section 3. The spatial discretization is second order accurate and uses a combination of centered Finite Differences and BDF [1], [5]. The semidiscretized in space system corresponds to a semi-explicit index 2 Differential Algebraic Equation (DAE). The time discretization is done by a linearly implicit Euler discretization, which, although only first order, is the simplest acceptable numerical approach for the above type of DAEs. Our numerical experiments show it to be both robust and efficient in the present context. Computational results are presented and discussed Section 4. Finally, Section 5 is devoted to concluding remarks.

\section{THE MODEL}

We consider general axisymmetric hoppers as illustrated in Figure 1. In order to simplify the problem, a pseudo one-dimensional formulation is derived by averaging all quantities on horizontal cross-sections. The height of the powder in the container at a generic time $t$ can thus be described by a function $H(t)$. Using cylindrical coordinates, the domain 
occupied by the powder at time $t$ is given by

$$
\{(r, \theta, z) ; 0<z \leq H(t), 0 \leq \theta \leq 2 \pi, 0 \leq r<R(z)\}
$$

where $R(z)$ stands for the radius of the hopper at height $z$.

For an arbitrary function $f$, we define

$$
\bar{f}(z)=\frac{1}{\pi R^{2}(z)} \int_{0}^{R(z)} \int_{0}^{2 \pi} f(r, \theta, z) r d r d \theta
$$

where $\bar{f}(z)$ is the averaged value of the function $f$ at height $z$ in the hopper. By axisymmetry, the functions considered here do not depend on the angular variable $\theta$, and thus with a slight abuse of notation

$$
\bar{f}(z)=\frac{2}{R^{2}(z)} \int_{0}^{R(z)} f(r, z) r d r .
$$

Invoking axisymmetry, the stress tensor has the form

$$
T=\left[\begin{array}{ccc}
\sigma_{r r} & 0 & \sigma_{r z} \\
0 & \sigma_{\theta \theta} & 0 \\
\sigma_{r z} & 0 & \sigma_{z z}
\end{array}\right]
$$

Consider an infinitesimal slice of material of height $\delta z$. The forces acting on such a slice are averaged and summed. The average vertical stress is clearly given by $\bar{\sigma}_{z z}$. We denote by $\gamma$ and $p$ the bulk density and gas pressure respectively, and by $\bar{\gamma}$ and $\bar{p}$, their respective average values. The various forces are

- weight of solid: $-\bar{\gamma} \pi R^{2}(z) \delta z$;

- if $\bar{\tau}_{w}$ is the average wall shear stress and $\bar{\sigma}_{w}$ the average normal stress on the wall, there are upward forces of $2 \pi R(z) \delta z \bar{\tau}_{w}$ and $2 \pi R(z) R^{\prime}(z) \delta z \bar{\sigma}_{w}$;

- upward pressure due to the wall: $2 \pi R(z) R^{\prime}(z) \delta z \bar{p}(z, t)$;

- pressure at bottom: $\bar{p}(z, t) \pi R^{2}(z)$, and top: $-(\bar{p}(z, t)+\delta p) \pi R^{2}(z+\delta z)$; this creates a force $-\pi R(z)^{2} \delta p-2 \pi R(z) R^{\prime}(z) \bar{p}(z, t) \delta z$;

- average vertical stress at bottom: $\bar{\sigma}_{z z}(z)$, and top: $-\left(\bar{\sigma}_{z z}(z)+\delta \sigma_{z z}\right)$; (compressive stresses are taken as positive for granular material); this creates a force $-\pi R^{2}(z) \delta \sigma_{z z}-$ $2 \pi R(z) R^{\prime}(z) \bar{\sigma}_{z z} \delta z$.

The resulting balance of forces equation is

$$
\partial_{z} \bar{\sigma}_{z z}+\partial_{z} \bar{p}+\frac{2 R^{\prime}(z)}{R(z)} \bar{\sigma}_{z z}-\frac{2}{R(z)}\left(\bar{\tau}_{w}+\bar{\sigma}_{w} R^{\prime}(z)\right)+\bar{\gamma}=0
$$

On the wall, the law of sliding friction applies

$$
\bar{\tau}_{w}=\mu_{w} \bar{\sigma}_{w}
$$

where $\mu_{w}$ is the coefficient of wall friction. The average stress tensor $\bar{T}$ is diagonal; in other words, both $\bar{\sigma}_{r r}$ and $\bar{\sigma}_{z z}$ are principal while $\bar{\sigma}_{r z}=0$. Notice that $\bar{\sigma}_{w}(z)=$ 
$\cos ^{2} \theta(z) \sigma_{r r}(z)+\sin ^{2} \theta(z) \sigma_{z z}(z)$ where $\tan \theta(z)=R^{\prime}(z)$. The ratio of average vertical stress $\bar{\sigma}_{z z}$ to average wall stress $\bar{\sigma}_{w}$

$$
k=\frac{\bar{\sigma}_{w}}{\bar{\sigma}_{z z}}
$$

is taken as depending on the geometry only, see $\S 4$ for more details. The above assumption is the pendant to Janssen's analysis, [6], [8], [10], which is routinely used in vertical bunkers. In (3), $k$ depends on the geometry of the container and has to be determined through experiments [8], [13] and $\S 4$. Equations (2) and (3) then yield

$$
\partial_{z} \bar{\sigma}_{z z}+\partial_{z} \bar{p}+\frac{2 R^{\prime}(z)}{R(z)} \bar{\sigma}_{z z}-\frac{2}{R(z)}\left(\mu_{w}+R^{\prime}(z)\right) k \bar{\sigma}_{z z}+\bar{\gamma}=0 .
$$

Let $\Gamma$ be the density of the solid particles, which is assumed constant. The gas density, denoted by $\rho$, is an unknown function of time and position. These two quantities are linked through the bulk density $\gamma$

$$
\gamma=f_{s} \Gamma+\left(1-f_{s}\right) \rho
$$

where $f_{s}$ is the volume fraction occupied by the solid. Generally $\Gamma$ is at least three orders of magnitude larger that $\rho$, and thus

$$
f_{s} \approx \frac{\gamma}{\Gamma}
$$

The average bulk density is considered as a function of the average major consolidating stress, here $\bar{\sigma}_{z z}$, i.e., $\bar{\gamma}=\bar{\gamma}\left(\bar{\sigma}_{z z}\right)$. Various such relations have been proposed, see [10], $\S 6.2$, for a review. Those models typically make sense for only a limited range of values of $\bar{\sigma}_{z z}$. Following [7], we assume

$$
\bar{\gamma}=\gamma_{m}\left(1+\frac{\bar{\sigma}_{z z}}{\sigma_{m}}\right)^{\beta}
$$

where $0 \leq \beta<1$ is the coefficient of compressibility of the material, $\gamma_{m}>0$ and $\sigma_{m}>0$ are material constants. We refer to [3] and [2] for respectively theoretical and experimental investigations of the precise nature of the bulk density/stress relation.

Assuming powder is neither entering nor leaving the system, the total mass $M$ of the solid is conserved, leading to

$$
\int_{0}^{H(t)} R^{2}(z) \bar{\gamma}(\bar{\sigma}(z, t)) d z=\frac{M}{\pi} \equiv \tilde{M}, \quad t \geq 0 .
$$

Applying the continuity equation to both gas and solid phases, we get

$$
\begin{array}{r}
\partial_{t} \gamma+\nabla \cdot\left(\gamma \mathbf{u}_{\mathbf{s}}\right)=0 \\
\partial_{t}\left(\left(1-\frac{\gamma}{\Gamma}\right) \rho\right)+\nabla \cdot\left(\left(1-\frac{\gamma}{\Gamma}\right) \rho \mathbf{u}_{\mathbf{g}}\right)=0
\end{array}
$$

where $\mathbf{u}_{\mathbf{s}}$ and $\mathbf{u}_{\mathbf{g}}$ are the velocities of the solid and the gas, respectively. 
Using axial symmetry and applying the averaging operator (1) to (9) yields

$$
\partial_{t} \bar{\gamma}+\frac{2}{R(z)} \gamma(R(z), z) u_{s, r}(R(z), z)+\frac{2}{R(z)^{2}} \int_{0}^{R(z)} \partial_{z}\left(\gamma(r, z) u_{s, z}(r, z)\right) r d r=0
$$

Further, assuming the grains in contact with the wall to move tangentially with respect to it, we observe $u_{s, r}(R(z), z)=R^{\prime}(z) u_{s, z}(R(z), z)$. Elementary manipulations then leads to

$$
\partial_{t} \bar{\gamma}+\frac{1}{R^{2}(z)} \partial_{z}\left(R^{2}(z) \overline{\gamma u_{s, z}}\right)=0
$$

Neglecting fluctuations in the radial direction gives $\overline{\gamma u_{s, z}} \approx \bar{\gamma} \overline{u_{s, z}}$. Similar principles can be applied to local conservation of gas. Those conservation laws then read

$$
\begin{aligned}
\partial_{t} \bar{\gamma}+\frac{1}{R^{2}(z)} \partial_{z}\left(R^{2}(z) \bar{\gamma} \bar{u}_{s, z}\right) & =0 \\
\partial_{t}\left(\left(1-\frac{\bar{\gamma}}{\Gamma}\right) \bar{\rho}\right)+\frac{1}{R^{2}(z)} \partial_{z}\left(R^{2}(z) \bar{\rho}\left(1-\frac{\bar{\gamma}}{\Gamma}\right) \bar{u}_{g, z}\right) & =0
\end{aligned}
$$

In addition to (3) and (7), two additional constitutive equations are considered. First, the gas is assumed to be ideal and isothermal

$$
\frac{p}{\rho}=\frac{p_{0}}{\rho_{0}}
$$

where $p_{0}$ and $\rho_{0}$ are constant reference values. Second, pressure gradient and velocities are related through Darcy's law, which reads here

$$
\bar{u}_{g, z}-\bar{u}_{s, z}=-K(\bar{\gamma}) \partial_{z} \bar{p}
$$

where $K$ is the permeability, taken as a function of the average bulk density. We take [8]

$$
K(\bar{\gamma})=K_{0}\left(\frac{\bar{\gamma}}{\gamma_{0}}\right)^{-a}
$$

where $K_{0}$ and $\gamma_{0}$ are reference values and $a$ is a positive constant. The parameters $\beta, \sigma_{m}$, $\gamma_{m}, a, K_{0}$ and $\gamma_{0}$ appearing in (7) and (15) have to be determined experimentally.

We now eliminate velocities from the system. Putting together (14) and (11) yields, after integration between 0 and a generic point $z$

$$
\begin{aligned}
\int_{0}^{z} R^{2}(\tilde{z}) \partial_{t} \bar{\gamma}(\tilde{z}) d \tilde{z} & +R^{2}(z) \bar{\gamma}(z)\left(\bar{u}_{g, z}(z)+K(\bar{\gamma}(z)) \partial_{z} \bar{p}(z)\right) \\
& -R^{2}(0) \bar{\gamma}(0)\left(\bar{u}_{g, z}(0)+K(\bar{\gamma}(0)) \partial_{z} \bar{p}(0)\right)=0
\end{aligned}
$$

Assuming no gas leaves the (closed) container at $z=0$, natural boundary conditions at the bottom are then $\bar{u}_{g, z}(0, t)=0$ and $\partial_{z} \bar{p}(0, t)=0$. The previous equation can be solved for $\bar{u}_{g, z}(z)$, leading to

$$
\bar{u}_{g, z}(z)=-\frac{1}{\bar{\gamma}(z) R^{2}(z)} \int_{0}^{z} R^{2}(\tilde{z}) \partial_{t} \bar{\gamma}(\tilde{z}) d \tilde{z}-K(\bar{\gamma}(z)) \partial_{z} \bar{p}(z)
$$


Using (13) and the above expression for $\bar{u}_{g, z}(z),(12)$ can be rewritten in terms of pressure $p$ eliminating $\rho$ from the problem.

The full system of equations can now be considered. The three unknowns of the problem are the averaged gas pressure $\bar{p}$, the averaged vertical stress $\bar{\sigma}_{z z}$ and the height of the powder $H(t)$. We obtain the following system of integrodifferential equations in $\{(z, t) ; 0<t, 0<z<H(t)\}$

$$
\begin{aligned}
0= & \left(1-\frac{\bar{\gamma}}{\Gamma}\right) \partial_{t} \bar{p}-\frac{\bar{p}}{\bar{\gamma}} \partial_{t} \bar{\gamma}-\frac{1}{R^{2}(z)} \partial_{z}\left(\bar{p}\left(\frac{1}{\bar{\gamma}}-\frac{1}{\Gamma}\right)\right) \int_{0}^{z} R^{2}(z) \partial_{t} \bar{\gamma} \\
& -\left(1-\frac{\bar{\gamma}}{\Gamma}\right) \partial_{z}\left(\bar{p} K \partial_{z} \bar{p}\right)+\frac{K \bar{p}}{\Gamma} \partial_{z} \bar{\gamma} \partial_{z} \bar{p} \\
0= & \partial_{z} \bar{\sigma}_{z z}+\partial_{z} \bar{p}+2 \frac{R^{\prime}(z)}{R(z)} \bar{\sigma}_{z z}-\frac{2}{R(z)}\left(\mu_{w}+R^{\prime}(z)\right) k \bar{\sigma}_{z z}+\bar{\gamma} \\
\tilde{M}= & \int_{0}^{H(t)} R^{2}(z) \bar{\gamma}\left(\bar{\sigma}_{z z}(\tilde{z}, t)\right) d \tilde{z}
\end{aligned}
$$

The system is completed by initial and boundary conditions

$$
\begin{aligned}
\bar{p}(\cdot, 0) & =p_{0}, \\
\bar{\sigma}_{z z}(H(t), t)=0, \quad \partial_{z} \bar{p}(0, t) & =0, \quad \bar{p}(H(t), t)=p_{a t m},
\end{aligned}
$$

where $p_{a t m}$ is the value of atmospheric pressure. The boundary condition at $z=0$ corresponds to a solid bottomed container. This can be easily modified to include the case when some material exits through an outlet at the bottom. A mathematical analysis of the above problem in the case of a cylindrical container, i.e., $R^{\prime}(z)=0$, can be found in [4].

A useful tool for the present kind of equations consists in mapping the entire problem into a fixed spatial domain. Upon discretization, this type of formulation leads to better stability properties than formulations where the grid moves with the material, such as [8]. Let

$$
y=z / H(t) \quad p(y, t)=\bar{p}(z, t) \quad \sigma(y, t)=\bar{\sigma}_{z z}(z, t) .
$$

Based on these relations, the previous system of equations (16), (17) and (18) yields

$$
\begin{aligned}
0 & =\left(1-\frac{\gamma}{\Gamma}\right)\left(\partial_{t} p-y \partial_{y} p \frac{H^{\prime}(t)}{H(t)}\right)-\frac{p}{\gamma}\left(\gamma^{\prime} \partial_{t} \sigma-y \gamma^{\prime} \partial_{y} \sigma \frac{H^{\prime}(t)}{H(t)}\right) \\
& -\frac{1}{R^{2}(H(t) y)} \partial_{y}\left(p\left(\frac{1}{\gamma}-\frac{1}{\Gamma}\right)\right) \int_{0}^{y} R^{2}(H(t) y)\left(\gamma^{\prime} \partial_{t} \sigma-y \gamma^{\prime} \partial_{y} \sigma \frac{H^{\prime}(t)}{H(t)}\right) \\
& -\frac{1}{H^{2}(t)}\left(1-\frac{\gamma}{\Gamma}\right) \partial_{y}\left(p K \partial_{y} p\right)+\frac{K p}{\Gamma H^{2}(t)} \gamma^{\prime} \partial_{y} \sigma \partial_{y} p \\
0 & \left.=\partial_{y} \sigma+\partial_{y} p+2 \frac{R^{\prime}(H(t) y) H(t)}{R(H(t) y)} \sigma-\frac{2 H(t)}{R(H(t) y)}\left(\mu_{w}+R^{\prime}(z)\right) k \sigma+H(t) \gamma,(20)\right) \\
\tilde{M} & =H(t) \int_{0}^{1} R^{2}(H(t) y) \gamma(\sigma(y, t)) d y
\end{aligned}
$$

where we have dropped the bar notation. Initial and boundary conditions become

$$
\begin{aligned}
p(y, 0) & =p_{0}(y), \quad y \in(0,1), \\
\sigma(1, t)=0, \quad \partial_{y} p(0, t) & =0, \quad p(1, t)=p_{a t m},
\end{aligned}
$$


The change from $R(z)=c s t$ to a general function $R(z)$ is far from being benign. If $R(z)=$ $R_{0}=c s t,(21)$ can be solved for $H(t): H(t)=\frac{\tilde{M}}{R_{0}^{2}}\left(\int_{0}^{1} \gamma(\sigma(y, t)) d y\right)^{-1}$. Taking the derivative, one can also obtain an explicit expression for $H^{\prime}(t)$. Those expressions can be then substituted in (19) and (20) to fully eliminate $H$ from the system [4]. For general functions $R$ however, (21) is a nonlinear equation for $H(t)$, which cannot be expected to always yield explicit solutions. To maintain generality, $H(t)$ is not eliminated from the system before discretization but is rather computed as an unknown along with stress and pressure.

\section{DISCRETIZATION}

These equations are discretized and solved in the fixed rectangular domain $(0,1) \times(0, T)$. Set $\Delta y=1 / N$ and let $y_{i}=(i-1) \Delta y \quad i=1, \ldots, N+1$. The semidiscretized variables are pressure $P(t)=\left[P_{1}(t), \ldots, P_{N}(t)\right]$ and stress $\Sigma(t)=\left[\Sigma_{1}(t), \ldots, \Sigma_{N}(t)\right]$. Note that the remaining unknown, $H$, depends only on time. The boundary conditions at $y=1$ read $P_{N+1}=P_{a t m}$ and $\Sigma_{N+1}=0$. A second order spatial discretization for both variables is used. The discretized operators are

$C_{p}: N \times N$ matrix corresponding to a second order centered discretization of $\partial_{y}$ with pressure boundary conditions (at $y=0$ and $y=1$ ),

$D: N \times N$ matrix corresponding to a second order centered discretization of $\partial_{y y}$ with pressure boundary conditions (at $y=0$ and $y=1$ ),

$C_{\sigma}: N \times N$ matrix corresponding to a second order backward differentiation formula (BDF [1], [5]) for $\partial_{y}$ with stress boundary condition (at $y=1$ ).

Although more sophisticated discretization methods, such as BDF based algorithms [1], should be considered in space as well in general, the diffusion like dynamics of the present problem are sufficiently simple to mitigate this point of view. The construction of $C_{p}$ and $D$ is elementary. For $C_{\sigma}$ we take

$$
C_{\sigma}=\frac{1}{2 \Delta x}\left[\begin{array}{rrrrrrr}
-3 & 4 & -1 & 0 & \ldots & \ldots & 0 \\
0 & -3 & 4 & -1 & 0 & \ldots & 0 \\
\ldots & \ldots & \ldots & \ldots & \ldots & \ldots & \ldots \\
0 & \ldots & 0 & -3 & 4 & -1 & 0 \\
0 & \ldots & \ldots & 0 & -3 & 4 & -1 \\
0 & \ldots & \ldots & \ldots & 0 & -3 & 4 \\
0 & \ldots & \ldots & \ldots & \ldots & 0 & -2
\end{array}\right] .
$$

In addition, the following discretized integral operators are introduced

$$
A_{\Delta}(W)=\frac{1}{\tilde{M}} \sum_{j=1}^{N+1} \omega_{j} W_{j} \quad \text { and }\left(B_{\Delta}(W)\right)_{i}=\sum_{j=1}^{i} \omega_{j} W_{j}
$$

where $\omega$ denotes the weights of the quadrature. Three additional variables corresponding to the derivatives of the three main three unknowns $P, \Sigma$ and $H$ are also introduced, $U=d_{t} P$, $V=d_{t} \Sigma$ and $L=H^{\prime}$. The spatially discretized problem is

$$
d_{t} P=U
$$




$$
\begin{aligned}
0 & =\left(1-\frac{\gamma}{\Gamma}\right)\left(U-\frac{L}{H} Y C_{p} P\right)-\frac{P}{\gamma}\left(\gamma^{\prime} V-\gamma^{\prime} \frac{L}{H} Y C_{\sigma} \Sigma\right) \\
& -\frac{1}{R^{2}}\left(\left(\frac{1}{\gamma}-\frac{1}{\Gamma}\right) C_{p} P-\frac{P \gamma^{\prime}}{\gamma^{2}} C_{\sigma} \Sigma\right)\left(B_{\Delta}\left(R^{2} \gamma^{\prime} V\right)-\frac{L}{H} B_{\Delta}\left(R^{2} \gamma^{\prime} Y C_{\sigma} \Sigma\right)\right) \\
& -\frac{1}{H^{2}}\left(1-\frac{\gamma}{\Gamma}\right) D(P, \Sigma) P+\frac{P K}{\Gamma H^{2}} \gamma^{\prime} C_{\sigma} \Sigma C_{P} P+b c 1 \equiv f(P, U, V, \Sigma, H, L) \\
0 & =C_{\sigma} \Sigma+C_{p} P+\frac{2 H R^{\prime}}{R(H Y)} \Sigma+b c 2-\frac{2 H}{R(H Y)}\left(\mu_{w}+R^{\prime}\right)+H \gamma \\
& \equiv G(P, U, V, \Sigma, H, L) \\
d_{t} \Sigma & =V \\
d_{t} H & =L \\
0 & =H A_{\Delta}\left(R^{2}(H Y) \gamma\right) \equiv h(P, U, V, \Sigma, H, L)
\end{aligned}
$$

where $\gamma(\Sigma)$ and $\gamma^{\prime}(\Sigma)$ are vectors and $Y=\left[y_{1}, \ldots, y_{N}\right]$. The two vectors $b c 1$ and $b c 2$ result from the presence of boundary conditions. Also, vector to vector multiplication in the above expressions is taken to be componentwise. The problem has the form

$$
\begin{aligned}
d_{t} P & =U, \\
0 & =f(P, U, V, \Sigma, H, L), \\
0 & =g(P, 0,0, \Sigma, H, 0), \\
d_{t} \Sigma & =V \\
d_{t} H & =L \\
0 & =h(0,0,0, \Sigma, H, 0)
\end{aligned}
$$

This corresponds to an semi-explicit index 2 DAE or equivalently a fully implicit index 1 DAE [1]. A linearly Implicit Euler time discretization [5], [11] of (24)-(29) leads

$$
\left[\begin{array}{cccccc}
I & -\Delta t I & 0 & 0 & 0 & 0 \\
-\Delta t f_{1}^{n} & -\Delta t f_{2}^{n} & -\Delta t f_{3}^{n} & -\Delta t f_{4}^{n} & -\Delta t f_{5}^{n} & -\Delta t f_{6}^{n} \\
-\Delta t g_{1}^{n} & -\Delta t g_{2}^{n} & -\Delta t g_{3}^{n} & -\Delta t g_{4}^{n} & -\Delta t g_{5}^{n} & -\Delta t g_{6}^{n} \\
0 & 0 & -\Delta t I & I & 0 & 0 \\
0 & 0 & 0 & 0 & 1 & -\Delta t \\
-\Delta t h_{1}^{n} & -\Delta t h_{2}^{n} & -\Delta t h_{3}^{n} & -\Delta t h_{4}^{n} & -\Delta t h_{5}^{n} & -\Delta t h_{6}^{n}
\end{array}\right]\left[\begin{array}{c}
P^{n+1}-P^{n} \\
U^{n+1}-U^{n} \\
V^{n+1}-V^{n} \\
\Sigma^{n+1}-\Sigma^{n} \\
H^{n+1}-H^{n} \\
L^{n+1}-L^{n}
\end{array}\right]=\Delta t\left[\begin{array}{c}
U^{n} \\
f^{n} \\
g^{n} \\
V^{n} \\
L^{n} \\
h^{n}
\end{array}\right]
$$

where the subscripts denote derivatives with respect to the corresponding variables. The first partial derivatives of $f$ and $g$ are evaluated through finite differences.

\section{COMPUTATIONAL RESULTS}

Two cases with different geometries are considered. The first corresponds to a cylinder and the second to a bunker consisting of a cylinder for the upper portion and a cone for the lower portion. The data is initialized as follows.

The values of various parameters in the model have been taken as to represent a realistic situation

$$
\begin{array}{ll}
\beta_{m}=.25 & \Gamma=200 \mathrm{lbs} / \mathrm{ft}^{3} \\
\gamma_{m}=60 \mathrm{lbs} / \mathrm{ft}^{3} & K_{0}=10^{-4} \mathrm{ft}^{4} \mathrm{lbs}^{-1} \mathrm{~s}^{-1} \\
\sigma_{m}=13 \mathrm{lbs} / \mathrm{ft}^{2} & a=4 \\
\gamma_{0}=80 \mathrm{lbs} / \mathrm{ft}^{3} & \mu_{w}=\tan (\pi / 9)
\end{array}
$$


The atmospheric pressure is $p_{a t m}=2116.8 \mathrm{lbs} / \mathrm{ft}^{2}$.

The determination of the ratio $k$ between wall stress and vertical stress, see (3), is more delicate. In the case of a perfectly plastic flow, such a coefficient could be derived from a plasticity model, Mohr-Coulomb for instance [10]. Although this is often done as part of an analysis à la Janssen, such an approach is not consistent with the mechanical states considered here since one cannot assume the material to have reached yield. The value of the ratio $k$ does, however, depend on the geometry. For plastic granular flows, the stresses are usually believed to be close to an active state in a vertical cylindrical hopper, while they tend to be in a passive state in converging conical hoppers for instance [10], [15]. Experimental evidence [13] seems to point to the fact that, even here, a similar behavior can be observed. More precisely, the value of $k$ tends to be much lower in a converging conical section than in a vertical cylindrical one. The values below are taken to reflect this.

Let us consider the cylinder with radius $R=0.5 \mathrm{ft}$ (and thus $R^{\prime}=0$ ) with an initial height of powder $H(0)=5 \mathrm{ft}$. The ratio $k$ is taken as $k=1 / 3$. An initial pressure field, $p_{0}$, consistent with the boundary conditions in (23) is given as

$$
p_{0}(z)=p_{a t m}\left(1+\frac{H(0)}{100}\left(1-\left(\frac{z}{H(0)}\right)^{2}\right)\right)
$$

In the calculations below, $N=200$ and $N T=T / \Delta t=500$.

For the second case, the radius of the cylinder is $R=0.5 \mathrm{ft}$. The radius at the bottom of the bunker is $0.4 \mathrm{ft}$, and the height of the conical part is $1 \mathrm{ft}$. Thus, $R^{\prime}=0.1$. In order to have approximately the same mass of material in this hopper as the first case, the total initial height of the powder is $H(0)=5.459 \mathrm{ft}$. The considered values of $k$ are $1 / 3$ in the cylindrical part, and $k=3$ in the conical part. The same initial pressure field is taken. In addition, $N=200$ and $N T=T / \Delta t=500$ as before.

Figure 2 gives a typical illustration of the behavior of the problem in a cylinder. Note that Figure 2 is related to the original geometry of the problem, i.e., the variable is $z$ not $y$. As expected, a settlement of the powder is observed. For both graphs, the top line corresponds to the position of the top of the powder column, i.e., the free boundary. Further, the pressure field is found to asymptotically converge to a uniform pressure value corresponding to $p_{a t m}$, the atmospheric pressure, i.e., equilibrium of the pressure is established. The stress field is also found to converge to a stationary distribution $\sigma_{\infty}$ which, in terms of the original variable $z$, is solution to

$$
\begin{gathered}
\partial_{z} \sigma_{\infty}-\frac{2}{R(z)} \mu_{w} k \sigma_{\infty}+\gamma\left(\sigma_{\infty}\right)=0, \quad 0<z<H_{\infty} \\
\sigma_{\infty}\left(H_{\infty}\right)=0
\end{gathered}
$$

where $H_{\infty}$ is the asymptotic value of the height of the powder column. Figure 4, left, illustrates the convergence to the asymptotic stationary state after 2000s.

Figure 3 gives a typical illustration of the behavior of the problem in a bunker consisting of a cylinder as the upper part and a cone in the lower part. Again settlement of the powder is observed by considering the top line of the graphs. As in the previous case, the pressure field asymptotically converges to a uniform pressure value corresponding to $p_{a t m}$. The stress field also converges to a stationary distribution $\sigma_{\infty}$ which, in terms of the original 

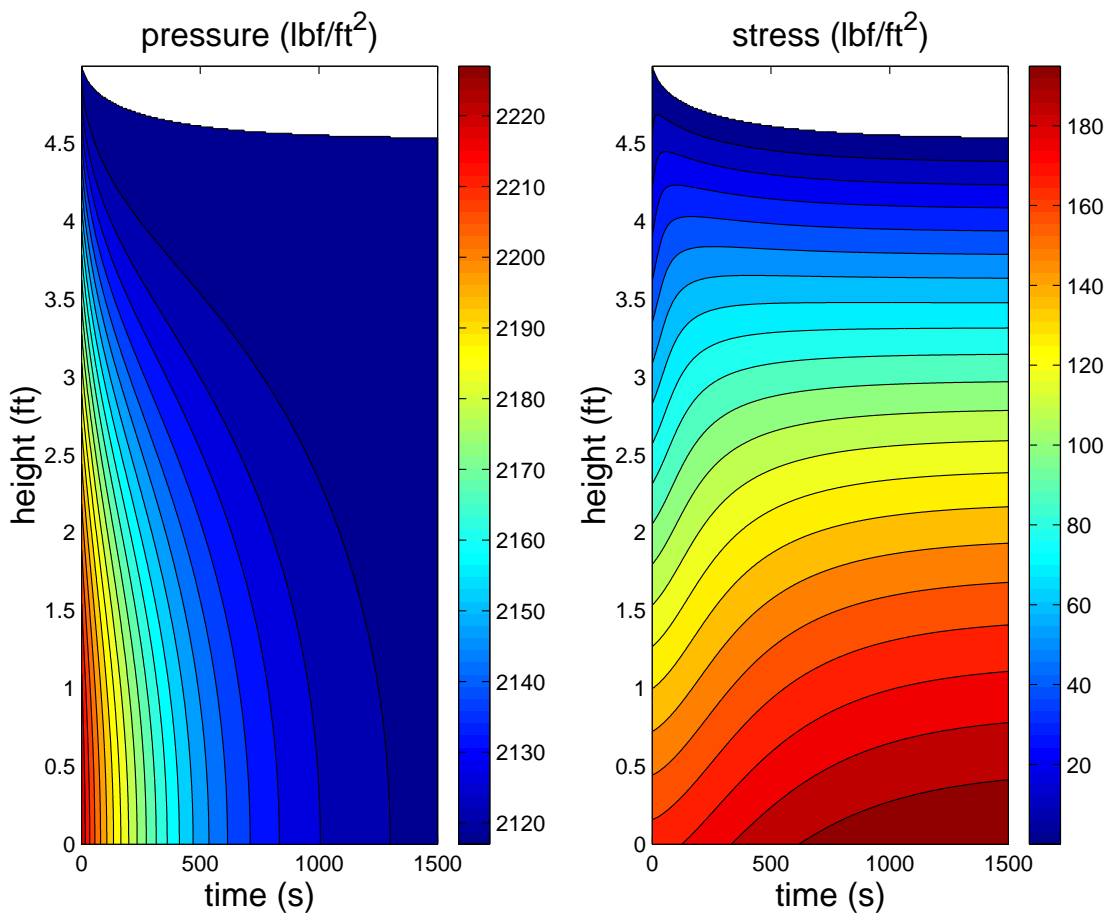

FIG. 2. Calculated pressure and stress fields in the cylindrical geometry; $N=200, N T=500, T=1500$.
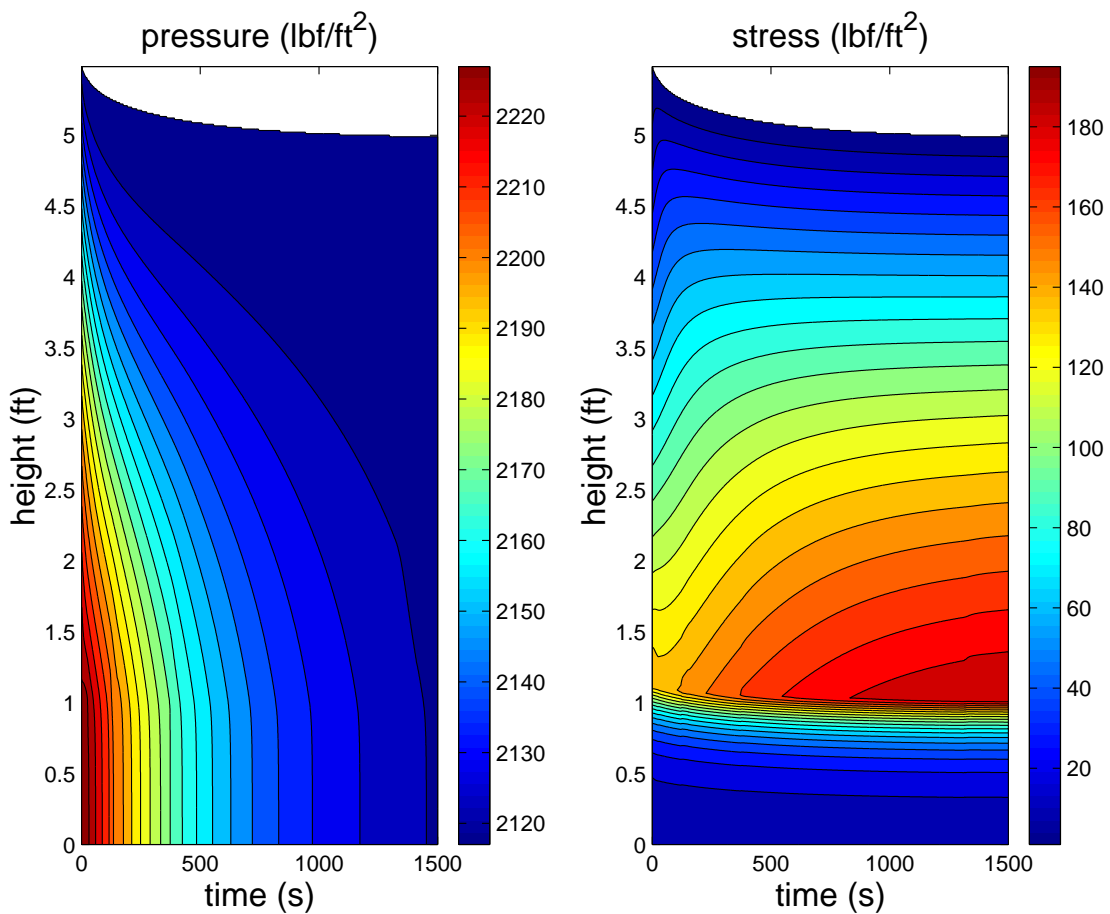

FIG. 3. Calculated pressure and stress fields in the cylinder-on-a-cone geometry; $N=200, N T=500$, $T=1500 \mathrm{~s}$. 

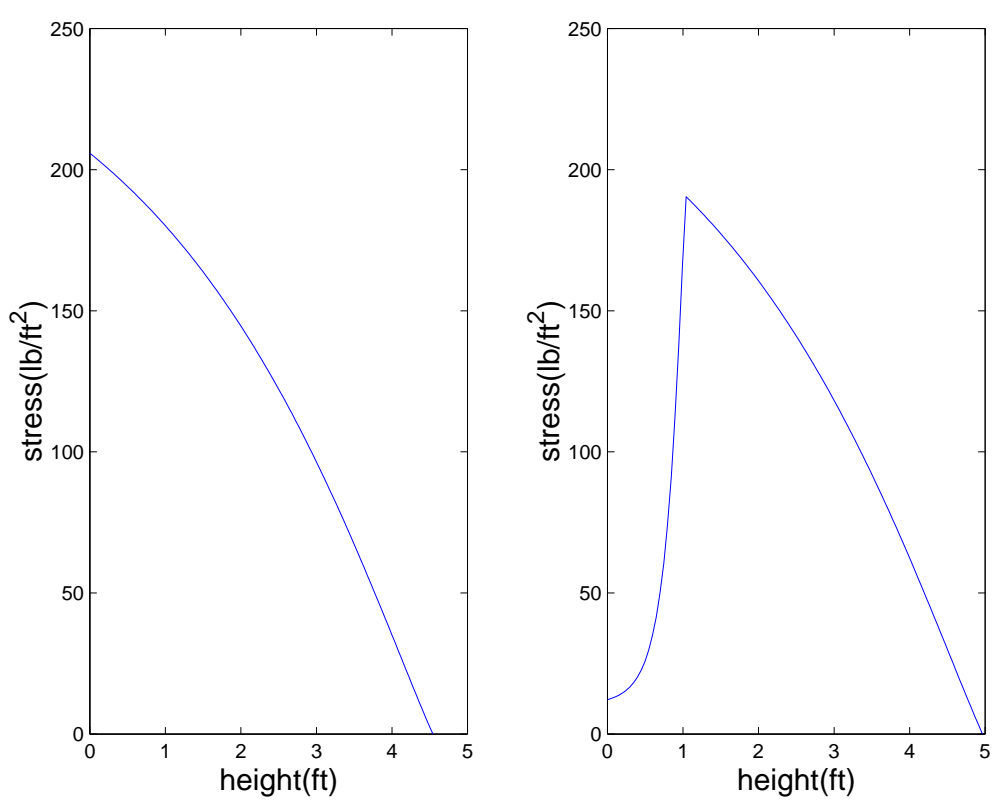

FIG. 4. Calculated stress field in the original geometry; left: cylinder, right:cylinder/cone geometry.

variable $z$, is solution to

$$
\begin{gathered}
\partial_{z} \sigma_{\infty}+2 \frac{R^{\prime}}{R(z)} \sigma_{\infty}-\frac{2}{R(z)}\left(\mu_{w}+R^{\prime}\right) k \sigma_{\infty}+\gamma\left(\sigma_{\infty}\right)=0, \quad 0<z<H_{\infty} \\
\sigma_{\infty}\left(H_{\infty}\right)=0
\end{gathered}
$$

where $H_{\infty}$ is the asymptotic value of the height of the powder column. Figure 4 illustrates the convergence to the asymptotic stationary state after 2000 s.

To verify the results of the calculations, an experiment must be designed so that the parameters for the model can be determined. Very accurate measurements of these parameters are needed to verify the calculated results with experimental ones. Consider Figure 5 which shows the half-life of the over-pressure at the bottom of a cylindrical bunker versus the compressibility parameter $\beta$. The half-lives range from nearly zero to approximately 650 for $\beta$ ranging from zero to four. It can be shown that the half-life of the over-pressure is also sensitive to other parameters in the model [12].

\section{CONCLUSIONS}

Existing models for powder consolidation have been extended to general axisymmetric domains in order to take into account geometrical effects. In this process and as with those prior models, averaging on horizontal cross sections plays a fundamental simplifying role. The resulting system consists of an essentially parabolic PDE, and an ODE in space and an integral equation. In spite of the strong nonlinear coupling, those can be thought of as respectively equations for the gas pressure, the vertical stress and the height of the powder. A fundamental and problematic material parameter is the ratio $k$ between wall stress and vertical stress.

Unfortunately, some of the parameters entering the model, such as $k$ or the compressibility $\beta$, are not easy to measure in a reliable way. To make matters worse, the present work 


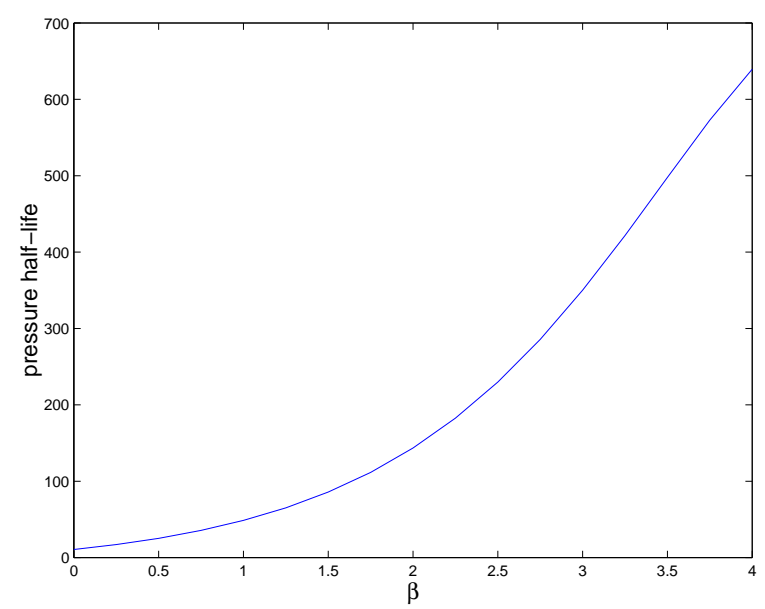

FIG. 5. Calculated half-life of over-pressure versus the parameter $\beta$.

illustrates that even relatively small variations in those coefficients can have a noticeable effect on the solution, see, e.g., Figure 5. This may partially explain the relative sparsity of experimental results for the present type of problems.

The main contributions of the paper are first a careful generalization of earlier models in order to take geometrical effects into account and second the design and implementation of a robust and efficient numerical algorithm for the corresponding equations. While they extend earlier results [4], [8], [12], the presented computational experiments seem to be consistent with them.

Various aspects of the present model are questionable, primarily the use of a pseudo onedimensional formulation through spatial averaging. Although a truly multidimensional approach appears to be desirable, its precise nature, especially in term of constitutive assumptions, is unclear.

\section{ACKNOWLEDGMENTS}

The authors are also grateful to Steve Campbell, Tim Kelley and Tony Royal for helpful discussions.

\section{REFERENCES}

1. K.E. Brenan, S.L. Campbell and L.R. Petzold, Numerical Solution of Initial-Value Problems in Differential-Algebraic Equations, SIAM, Classics in Applied Mathematics, \#14, 1996.

2. J. Duffy And R.D. Mindlin, Stress-strain relations and vibrations of a granular medium, J. Appl. Mech. (AMSE), 24 (1957), pp. 585-593.

3. J.D. Goddard, Nonlinear elasticity and pressure-dependent wave speeds in granular media, Proc. R. Soc. Lond. A, 430 (1990), pp.105-131.

4. P.A. Gremaud, C.T. Kelley, T.A. Royal and K.A. Coffey, On a powder consolidation problem, Technical report NCSU-CRSC-TROO-03, to be published in SIAM J. Appl. Math.

5. E. HAIRER AND G. WANNER, Solving ordinary differential equations II, stiff and differential-algebraic equations, 2nd Edition, Springer Verlag, 1991.

6. H.A. JAnssen, Versuche über Getreidedruck in Silozellen, Zeitschrift des Vereines Deutscher Ingenieure, 39 (1895), pp. 1045-1049.

7. A.W. JenIKE, Gravity flow of bulk solids, Bulletin No. 108, Utah Engineering Experiment Station, Salt Lake City, UT, 1961. 
8. J.R. Johanson And A.W. Jenike, The effect of gaseous phase on pressures in a cylindrical silo, Powder Tech., 5 (1971), pp. 133-145.

9. P.G. MurfitT AND P.L. Bransby, Deaeration of powders in hoppers, Powder Tech., 27 (1980), pp. 149162.

10. R.M. Nedderman, Static and kinematic of granular materials, Cambridge University Press, 1992.

11. L.R. Petzold, Order results for implicit Runge-Kutta methods applied to differential/algebraic systems, SIAM J. Numer. Anal., 23 (1986), pp. 837-852.

12. T. Rathbone, R.M. Nedderman and J.F. Davidson, Aeration, dearation, and flooding of fine particles, Chem. Engng. Sci., 42 (1987), pp. 725-736.

13. T.A. Royal, Private communication, Jenike \& Johanson, inc., 2000.

14. K. Terzaghi, Theoretical soil mechanics, Wiley, 1943.

15. D.M. WALKER, An approximate theory for pressures and arching hoppers, Chem. Engng. Sci., 21 (1966), pp. $975-997$.

16. J.K. Walters, A theoretical analysis of stresses in axially-symmetric hoppers and bunkers, Chem. Engng. Sci., 28 (1973), pp. 779-789. 